\title{
The increase of flexible protective materials strength characteristics by electrophysical influences
}

\author{
Irina Zlobina ${ }^{1, *}$, and Nikolai Bekrenev ${ }^{1}$ \\ ${ }^{1}$ Yuri Gagarin Saratov State Technical University, 410054 Saratov, Russia
}

\begin{abstract}
We studied the strength of the individual threads in the longitudinal and transverse rupture, the state of threads, as well as the strength of a single layer of aramid fabric exposed to the piercing influence of the conical indenter. Materials used in the experiments: special high molecular material of the brand DZh (SHMM-DZh) and ultra-high molecular weight polyethylene (UHMWP). It is found that the effect of electromagnetic field on the aramid threads and fabrics based on them, as well as the threads made of UMWP increases the tensile strength at 2 and 1.6-1.7 times respectively. Aramid fabric puncture resistance in the delivery state and in the wet state, after exposure to the electromagnetic field increases by 1.7-1.8 times. A significant reduction of the fiber bundle processed in the electromagnetic field of aramid fibers and UHMWP after exposure to tensile load was ascertained.
\end{abstract}

\section{Introduction}

The analysis of scientific literature, materials of conferences and exhibitions testifies the intensive development of the production of composite materials based on carbon fibers and glass fabrics $[1,2]$. Aramid threads in comparison with carbon and glass fibers are less fragile and suitable for processing on conventional equipment of textile manufactures [3, 4].

Research in the field of increasing the strength of such fabrics is aimed at improving technologies for the production of the starting components and the formation of tissue by optimizing the weaving [5], which makes the process difficult and expensive, and also leads to the need for adjustment or re-production, which lengthens the cycle of introducing the new developments.

Other promising material, used in protective equipment, both independently and as internal support for ceramic armor plates - UMWP, formed from woven fibers [6-8].

*Corresponding author: irinka_7@mail.ru 
Previously, we have obtained positive results in increasing the strength of structural composite materials based on a polymer matrix reinforced with carbon fibers, after electrophysical effects, in particular, an electromagnetic field of a certain frequency and intensity $[9,10]$.

The purpose of the research is to study the possibility of improving the performance properties, namely, strength of aramid and UHMWP materials when exposed to an electromagnetic field to improve their safety performance in extreme conditions, adjoint with a mechanical effect on the human.

\section{Materials and methods}

Experiments were carried out on fabrics and threads (SHMM-DZh) and UHMWPE threads. Tissue samples TSVM-G were tested for puncture in dry and wet condition. Threads of both types of materials were tested for rupture. In all the experiments on investigation of the physical and mechanical properties one-layer SHMM-DZh tissue samples of $20 \times 30$ $\mathrm{mm}$ and threads $0.05 \mathrm{~mm}$ thick and $70 \mathrm{~mm}$ long were used.

Special equipment developed by us enables testing of samples puncture and tear. In the experiments a concentrated stream of low energy, medium and high intensity was generated. In all experiments, the processing time was 1, 4 and 8 minutes. 3 samples were processed at each intensity level. With a cone angle of the 900 loading device we influenced the tissue measuring the current force is taken from the load cell. The puncture moment was fixed by the load torque chart's drawdown. The tests were conducted for dry and wet samples.

During electrophysical processing of the SHMM-DZh thread medium intensity mode was used, and low intensity mode for the UHMWP thread. The processing time took 4 minutes.

\section{Results and discussion}

Graphs showing the pressure increase kinetics until the moment of dry and wet samples puncture processed at an average intensity of electrophysical effects for 4 minutes are shown in Figure 1.

The research of the samples appearance after the puncture has showed that after electrophysical treatment both dry and wet samples puncture field form had a pronounced conical imprint, while in the control wet sample the loss of form was clearly noticeable, indicating the sliding and displacement of fibers, i.e. violation of their original weaving.

Analysis of the graphs (Figure 1) led to the conclusion that both wet control and processed samples have reduced at 1.5 times the puncture pressure compared with the dry sample. However, the wet sample, previously processed has $1.7-1.8$ times higher pressure puncture strength compared to the control one. Moreover, its strength is even 1.25 times higher than the control sample in the delivery condition. Tests of SHMM-DZh and UHMWP threads showed the following. Load increase kinetics of the sample with load applying to the SHMM-DZh thread in the axial direction is shown in Figure 2, and the UHMWP threads - in Figure 3.

The graphs show the different nature of the destruction of threads made of the studied materials. Aramid threads tear almost immediately, the graphics are of a smooth shape. UHMWP threads tear' charts have serrate character and show periodic load's bursts and falls. Analysis of the graphs shows that electrophysical processing at the average intensity of the impact increases the axial tensile load on the SHMM-DZh thread almost in 2 times. The influence of electrophysical processing on the thread's strength is significantly less 
pronounced in the cross direction. The tensile strength in the transverse direction does not increase by more than $18-20 \%$.

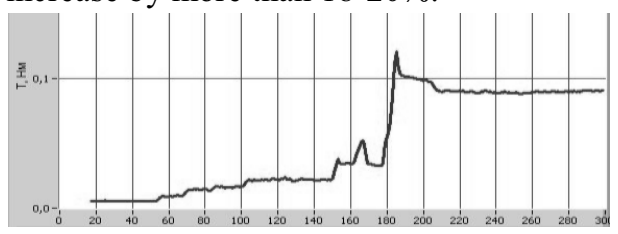

$a$

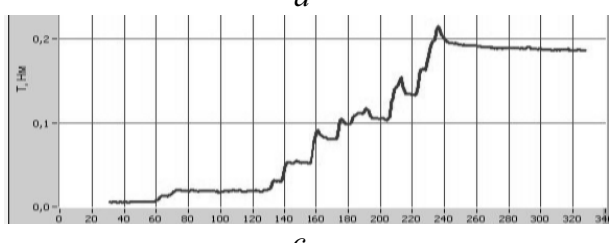

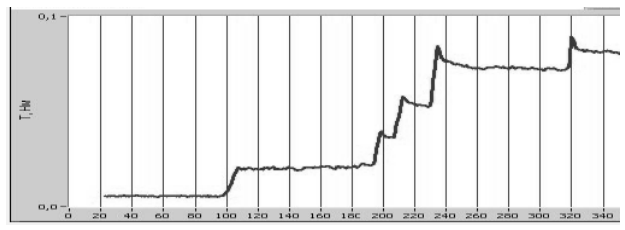

$b$

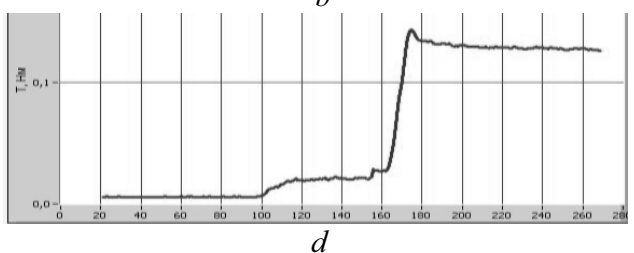

Fig. 1. Samples puncture effort' kinetics: control dry (a) and wet (b); dry processed (c) and wet (d).
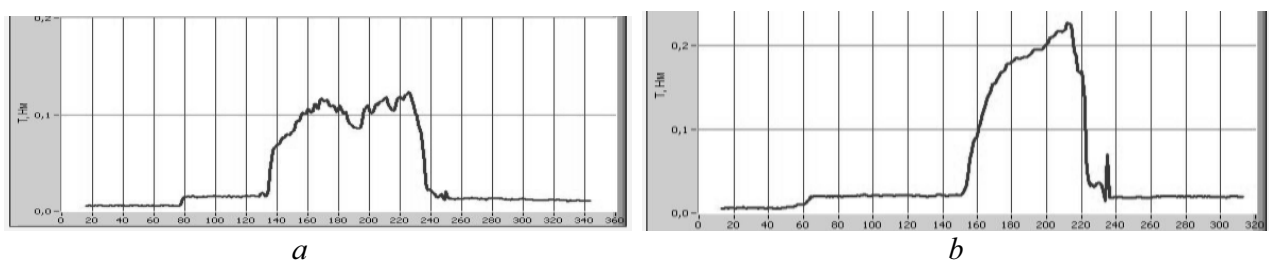

Fig. 2. The tests of the process of aramid thread SHMM-DZh axial gap kinetics: control sample (a); sample after electrophysical treatment (b).

Control thread UHMWP thread has bundle of constituent fibers after breaking. The fiber processed by electrophysical method has no significant bundles. $0.05 \mathrm{~mm}$ thick threads after processing have increased their tensile strength in 1.6-1.7 times, the threads $0.03 \mathrm{~mm}$ thick - nearly in 1.6 times. The threads elongation in $15-20 \mathrm{~mm}$ was noted. Decreasing the control threads thickness from $0.05 \mathrm{~mm}$ to $0.03 \mathrm{~mm}$ reduces the tear strength in $55 \%$, the processed thread - in $65 \%$.
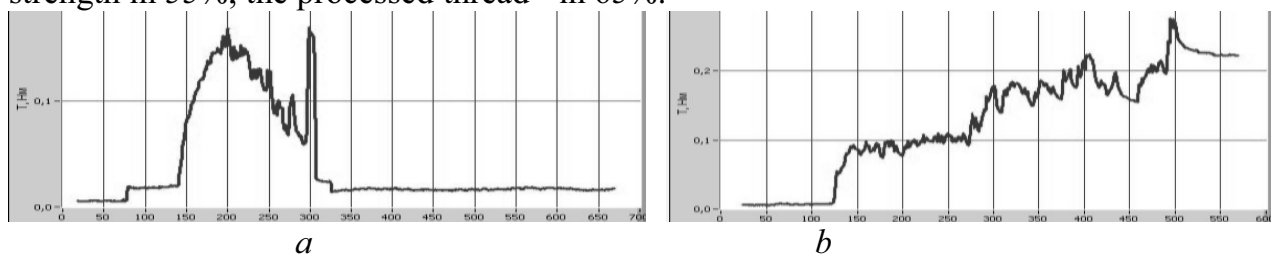

Fig. 3. The kinetics of the process of the axial gap filament UHMWP thickness of $0.05 \mathrm{~mm}$ control (a) and processed (b).

Based on the analysis of the results the following mechanism of improving the strength aramid fibers and tissues after exposure to electromagnetic fields can be offered. Studies [3, 4] has found that the structural units of the aramid fibers are rigid macromolecules or their aggregates - fibrils. High strength and stiffness of such fibers under tension are caused by a high degree of orientation of the macromolecules along the fiber axis and a high chemical bond dissociation energy. Stress state difference of neighboring structural elements causes an appearance of shear stresses in a boundary area between the fibers, which form the thread. As a result of uniaxial fiber's tensile is accompanied with germination of inter fibrillar cracks along the direction of the tensile force. However, the split fiber up to a certain limit is still in a state to percept the load, that is why Figure 3 (a) and (b) has a 
smooth character of increase to a certain meaning. Cleavage of threads apparently is accompanied by simultaneous breakage of individual the most stressed fiber fragments, after which the load is perceived by beam of fibrils and so on until the remaining fibers are unable to resist tensile load. A catastrophic complete destruction happens, accompanied by intensive splitting and fluffing of a sample. The impact of electromagnetic fields is likely to contribute to the growth of the number of links on inter - fibrillar macromolecular level, the organization of fibrils in the form of spirals, running along the fiber. Such spirals of the adjacent fibers may additionally communicate with each other at a macro level in the type «nut-screw» when a coil of a spiral enters the adjacent recess. This can happen at the initial time of loading, when the fibers are stretched and straightened and the gaps between the turns increase. With a further increase in load conditions of joint, rather than separate fiber work are formed. The occurrence of cracks that weakens the structure, mentioned above, that weakens the structure, is leveled in this case by the described action of the electromagnetic field generated in the spiral interfiber structures and additional fibrils bonds.

\section{Conclusions}

Short-term influence of electromagnetic field on the threads and fabric made of the materials used in the manufacture of flexible personal protective equipment such as UMWP and TSVM contributes both to increase tensile strength of the individual threads, and an increase in strength of the fabric when it is punctured. This reduces the dependence of the material on external factors, such as humidity. The reason for these results is the formation of additional zones tissue connections at micro level (fibrils and the fiber) and nanoscale (macromolecule) due to the interaction of the electromagnetic field with the macromolecules that form polymer tissue fiber, and of the fibers increase in elasticity. This improves the total tensile strength of threads and also increases the time period before the gap. Application processing in the electromagnetic field of the final elements made of aramid and UHMWP fabrics will improve the reliability and durability of aircraft crews personal protective equipment (PPE) for various purposes, as well as lung protective reinforced composite structures.

\section{References}

1. E. Kablov, Aviation materials and technology 1, 3 (2015)

2. E. Kablov, Herald of the Russian Academy of Sciences 82, 520 (2012)

3. Y. Shustov, A. Kurdenkova, Scientific and methodical electronic journal "Concept" 3, 36 (2016)

4. P. Shuldeshova, G. Zhelezina, Proceedings VIAM: electron. scientific-tech. Zh., 9 (2014)

5. P. Safonov, Razrabotka optimal'nyh tehnologicheskih parametrov izgotovlenija aramidnyh tkanej tehnicheskogo naznachenija// avtoref. diss. kand. tehn. nauk (2013)

6. E. Chistyakov, Tehnika i vooruzhenie 8, 1(2013)

7. A. Ignatova, A. Artemov, Basic Research 6-1, 101 (2012)

8. A. Bhatnagar, Lightweight ballistic composites (Woodhead Pub. Lim., Cambridge, 2006)

9. I. Zlobina, N. Bekrenev, Materials Science Forum 870, 101 (2016)

10. I. Zlobina, N. Bekrenev, High technologies 17-2, 25 (2016) 